\title{
Cooperation in the Commons with Unobservable Actions
}

\author{
by Nori Tarui, \\ Department of Economics, \\ University of Hawaii at Manoa \\ Charles Mason, \\ Department of Economics and Finance, \\ University of Wyoming \\ Stephen Polasky ${ }^{*}$, \\ Department of Applied Economics, \\ University of Minnesota \\ and Greg Ellis, \\ Department of Economics, \\ University of Washington
}

Working Paper No. 07-11

November 2006

\begin{abstract}
We model a dynamic common property resource game with unobservable actions and non-linear stock dependent costs. We propose a strategy profile that generates a worst perfect equilibrium in the punishment phase, thereby supporting cooperation under the widest set of conditions. We show under what set of parameter values for the discount rate, resource growth rate, harvest price, and the number of resource users, this strategy supports cooperation in the commons as a subgame perfect equilibrium. The strategy profile that we propose, which involves harsh punishment after a defection followed by forgiveness, is consistent with human behavior observed in experiments and common property resource case studies.
\end{abstract}

Key Words: Common property resource, cooperation, dynamic game, unobservable actions JEL Codes: D62, Q20

* Corresponding author: Department of Applied Economics, University of Minnesota, 1994 Buford Avenue, Saint Paul, MN 55108 USA, email: polasky@umn.edu, phone: (612)625-9213, fax: (612)6252729. 


\section{Introduction}

Since Gordon (1954), economists have known that individuals have incentives for excessive exploitation of common property resources. These incentives for excessive exploitation lead to the "tragedy of the commons" (Hardin, 1968). There are two potential externalities that lead to misguided incentives. Increased efforts by harvesters can impose crowding costs on other harvesters; this is at its core a static externality (Brown, 1974). This situation is quite similar to that facing individuals contributing to a public good. Indeed, one can think of the decision to reduce harvesting as a sort of contribution to the public good of "less crowding." There may also be a dynamic externality: larger current harvests reduce future resource stocks, which in turn may increase future harvest costs or constrain future harvest levels (Mason and Polasky, 1997). With either static or dynamic externalities, privately optimal behavior leads to socially excessive harvesting of a common property resource, yielding smaller welfare flows to society.

Despite the grim predictions of tragedy, a variety of researchers have found that many actual common-property harvesting regimes manage common property resources in a reasonably efficient manner, often for long periods of time, even with many agents involved (for reviews see Feeny, Hanna, and McEvoy, 1996; Ostrom 1990, 2000). The typical successful management regime has some means of limiting access to the commons and some means of punishment for over-harvesting. Access may be restricted to members of a particular community or group. Community members are responsible for monitoring and enforcement. Punishment can involve some type of loss of privilege, either temporary or permanent, or, for major offenses, banishment from the group.

A large number of experimental studies have investigated the common property problem, as well as the closely-related public goods provision problem (Ledyard, 1995). While 
many studies have found tendencies towards privately optimal behavior, several studies point to the possibility of more socially efficient outcomes. In one class of studies, subjects are able to identify specific players as defectors. Several papers show that when defectors can be singled out for punishment more socially efficient outcomes result (Casari and Plott, 2003; Fehr and Gachter, 2000a; Ostrom, Walker and Gardner, 1992). However, the observation of increased social efficiency does not rely solely on the ability to punish specific individuals who defect. Mason and Phillips (1997) study a game in which punishments cannot be tailored to the individual, but they still find that more socially efficient outcomes obtain. In a second class of studies, socially attractive outcomes occur even though subjects only know aggregate behavior (Cason and Khan, 1999; Chermak and Krause, 2002; Isaac and Walker, 1988; Hackett, Schlager and Walker, 1994; Ostrom, Gardner and Walker, 1994). In the majority of these studies, more cooperative outcomes are enhanced by communication. While several of these papers place subjects in a finitely-repeated game, some do not (Ostrom, Walker and Gardner, 1992; Mason and Phillips, 1997; Sadrieh and Verbon, 2005). These various empirical observations underscore the importance of understanding, from a conceptual point of view, how cooperative arrangements might take shape.

There has long been interest in examining the theoretical underpinnings of cooperative behavior. A rich literature in applied game theory has developed over the last two decades, evaluating the conditions under which equilibrium supports socially desirable outcomes in the presence of unilateral short-term incentives to deviate (Benhabib and Radner, 1992; Cave, 1987; Dutta, 1995a, 1995b; Dutta and Sundaram, 1993; Hannesson, 1997; Laukkanen, 2003; Polasky et al., 2006; Rustichini, 1992). The basic thrust of this literature has been to show that cooperation in the commons can be supported as a subgame perfect equilibrium, under certain 
conditions, through the use of strategies that include credible future punishment for deviations from the cooperative outcome. Dutta (1995a) proves that cooperation can be sustained for sufficiently patient players, an extension of the folk theorem result for repeated games. Several papers analyze dynamic resource games for arbitrary discount factors where payoffs are independent of stock (Benhabib and Radner, 1992; Cave, 1987; Dutta and Sundaram 1993; Rustichini, 1992). ${ }^{1} \quad$ While assuming stock independence is analytically convenient, this approach eliminates dynamic cost externalities important for a wide range of real-world common property resources. It also makes extraction to zero stock (extinction) profitable, which allows rapid extraction to extinction to be a credible punishment. Our analysis, like Hannesson (1997), Laukkanen (2003), and Polasky et al. (2006), explicitly considers the role of non-linear stock-dependent costs. We follow Clark (1973), and others, in using a discrete time model in which the marginal cost of harvest declines with increasing stock. When marginal cost declines in stock, there can be a strictly positive stock level that generates zero profits and below which further harvest generates negative marginal profit. In this case, credible punishment strategies are more complex, typically involving an on-going path of harvesting rather than rapid extinction. Another issue we address is imperfect monitoring of harvests. Punishing individual defectors is possible only if the resource users can identify who cheated. Polasky et al. (2006) analyze a game with costless monitoring and individual punishment schemes. However, monitoring is often prohibitively costly in the context of natural resource use. Ostrom (1990) suggests that, though monitoring is an important factor behind cooperative use of commons, cooperation is observed in some settings with imperfect monitoring, a finding in the experimental literature as well. In this paper, we assume that the resource stock level is observable but individual players' actions are not observable. When the resource stock falls

\footnotetext{
${ }^{1}$ Dutta (1995b) assumes that payoffs are linear in stock.
} 
below the cooperative level, what can be inferred is that someone cheated, but not who cheated. Therefore, punishment must be symmetric, targeting the whole group, rather than asymmetric, targeting a particular individual who cheated. Abreu et al. (1990) analyzed cooperation under imperfect monitoring in repeated games. This paper addresses cooperation under imperfect monitoring in a dynamic game where the stage game evolves endogenously given how the resource was used in previous periods.

Two prior papers, Hannesson (1997) and Laukkanen (2003), have addressed the issue of supporting cooperation in a common property resource with imperfect monitoring and non-linear stock dependent costs. Laukkanen (2003) analyzed cooperation in a two-player game in which fish stocks migrate between exclusive harvest zones so that players move sequentially. Cooperative solutions are supported by the threat to revert to non-cooperative Nash equilibrium if cheating is suspected. Hannesson (1997) uses a model similar to ours in that $N$ players move simultaneously in a discrete-time common property harvest game. Hannesson uses a simple punishment strategy in which players draw down the stock to the zero profit level of stock, where price equals marginal cost of harvest, in every period following a defection.

In contrast, we use a two-part punishment scheme, with heavy penalties in the first phase followed by eventual recovery of the stock to the cooperative level in the second phase. With a two-part punishment scheme, using the worst perfect equilibrium (i.e., a subgame perfect equilibrium with the lowest possible payoffs) following a defection offers the strongest possible incentive not to defect in the first place. In general, cooperation can be supported under a wider set of conditions by reversion to a worst perfect equilibrium rather than reversion to Nash equilibrium (Abreu et al., 1986; Abreu, 1988; Polasky et al., 2006). Moreover, with a two-part punishment scheme it need not be the case that cooperation is harder to support as the group size 
increases. We present a numerical simulation in section 5 that suggests cooperation could be easier to support with four players than with three. Our conjecture is that increases in group size have two conflicting effects. On the one hand, increases in group size reduce the share of the cooperative pie that any one player is allocated; all else equal this would tend to make cooperation harder to support. On the other hand, the set of potential punishers who must respond to an individual player that has cheated is larger when the group is larger. All else equal, this reduces the degree to which any one individual must increase its harvest to inflict the punishment, which tends to lower the cost associated with following through with the punishment. As such, larger punishments become credible, which tends to make cooperation easier to support. The numerical results we present below show that the combined impact of these two conflicting effects will be to make cooperation easier to support when group size is increased starting from relatively small values - at least in the context of our numerical example. Ultimately, however, the former effect dominates, and cooperation becomes more difficult to support as group size rises. These qualitative results are consistent with the experimental finding in Mason and Phillips (1997), who found more cooperative behavior with intermediate group sizes than with slightly smaller or slightly larger groups.

In the next section, we describe the dynamic common property resource game with unobservable actions and non-linear stock dependent costs. We characterize the cooperative outcome in section 3. In section 4, we analyze strategies designed to support cooperation as a subgame perfect equilibrium. We propose a two-part punishment scheme and demonstrate that, this two-part scheme can constitute a worst perfect equilibrium once a player has cheated, which then supports cooperation as a subgame perfect equilibrium under the widest possible set of circumstances. Section 5 contains a numerical illustration. Section 6 contains concluding 
comments.

\section{The Game}

We consider a discrete-time dynamic game with $N \geq 3$ players, indexed by $i$, who jointly harvest a common property renewable resource. ${ }^{2}$ In every period $t(=0,1, \ldots)$, each player $i$ chooses a harvest level, $h_{i t} \geq 0$. We assume that players choose harvest strategies in period $t$ simultaneously. Define total harvest in period $t$ as $h_{t}=\sum_{i=1}^{N} h_{i t}$. Let $s_{t}$ equal the amount of stock at the beginning of period $t$ and $z_{t}$ equal the stock at the end of period $t$ (escapement), with $z_{t}=s_{t}-h_{t}$. Between the end of period $t$ and the start of period $t+1$, stock grows according to a biological growth function: $s_{t+1}=g\left(z_{t}\right)$. We assume that the biological growth function, $g($.$) ,$ is a positive and strictly concave function for stock sizes between 0 and a positive carrying capacity $(K): g(0)=0, g(K)=K$, and $s<g(s), g^{\prime \prime}(s)<0$ for all $s \in(0, K)$. The initial stock $s_{0} \in(0, K]$ is given.

We assume that players observe stock at the beginning $\left(s_{t}\right)$ and end $\left(z_{t}\right)$ of each period so that they know aggregate harvest $\left(h_{t}\right)$, but that there is imperfect monitoring of harvests: player $i$ cannot observe $h_{j t}$ for $j \neq i$. Each player's action in period $t$ can depend on the initial level of stock in period $t\left(s_{t}\right)$ and the history of harvests through period $t-1$. Player $i$ 's strategy in period $t$ specifies $i$ 's action conditional on the information the player has in period $t$. We consider only pure strategies within a given period.

We assume that unit harvest cost depends upon stock size, $c(s)$. The unit harvest cost function $c$ is twice continuously differentiable, with $c(s)>0, c^{\prime}(s)<0, c^{\prime \prime}(s)>0$. We also

\footnotetext{
${ }^{2}$ Note that with $N=2$, the other player's harvest can be inferred from knowledge of one's own harvest and total harvest.
} 
assume that unit costs rise sufficiently rapidly as stock gets small so that $\int_{0}^{s} c(\omega) d w=\infty$ for any positive stock. Let $p>0$ be the exogenous price of harvest. For this to be an economically interesting problem, it must be worth harvesting at some stock level, and so we assume that $p>c(K)$. Define the zero profit level of stock as $\underline{s}: \quad p-c(\underline{s})=0$.

In any period, we assume that player $i$ receives a share of total returns equal to $i$ 's share of total harvest. Thus, player $i$ 's return in period $t$ is given by

$$
r_{i}\left(h_{1 t}, \ldots, h_{N t}, s_{t}\right)=\left\{\begin{array}{l}
\frac{h_{i t}}{h_{t}} \int_{s_{t}-h_{t}}^{s_{t}}[p-c(\omega)] d \omega, \text { for } h_{t} \leq s_{t} \\
-\infty, \text { for } h_{t}>s_{t} .
\end{array}\right.
$$

In essence, the assumption of equal sharing of returns is equivalent to assuming that the share of harvest for each player is constant throughout the time period. Alternatively, one could assume that all players harvest at the same rate during the time that they are active but that players harvesting more stock during the time period harvest for a longer amount of time. This alternative approach would weight harvest costs more heavily toward players that harvest greater amounts, i.e., that players with smaller shares earn higher profit per unit of harvest.

Each player's payoff for the entire game is given by the discounted sum of the period returns. We assume there is a common discount factor $\delta, 0<\delta<1$, by which payoffs are discounted from one period to the next.

\section{Characterizing cooperative outcome}

The joint-rent maximization problem is given by

$$
\max _{\left\{h_{t}\right\}_{t=0}^{\infty}} \sum_{t=0}^{\infty} \delta^{t} \int_{s_{t}-h_{t}}^{s_{t}}[p-c(\omega)] d \omega
$$




$$
\text { s.t. } \quad s_{t+1}=g\left(s_{t}-h_{t}\right), \quad h_{t} \geq 0, \quad s_{t} \geq 0, t=0,1, \ldots \text { given } s_{0} \in(0, K] \text {. }
$$

We can rewrite this problem as a dynamic programming problem focusing on escapement rather than harvest as the choice variable. The Bellman equation for this formulation is:

$$
V\left(s_{t}\right)=\max _{0 \leq z_{t} \leq s_{t}} \int_{z_{t}}^{s_{t}}[p-c(\omega)] d \omega+\delta V\left(g\left(z_{t}\right)\right)
$$

An optimal solution to the Bellman equation, which characterizes optimal escapement $\left(z^{*}\right)$ in every period with positive harvest, must satisfy

$$
-\left[p-c\left(z^{*}\right)\right]+\delta g^{\prime}\left(z^{*}\right) V^{\prime}\left(g\left(z^{*}\right)\right)=0,
$$

where $V^{\prime}\left(g\left(z^{*}\right)\right)=\left[p-c\left(g\left(z^{*}\right)\right)\right]$. Such $z^{*}$ exists under the assumptions on $c$ and $g$. We maintain the following assumption throughout this paper.

Assumption 1. $\frac{\partial}{\partial s}\left(-(p-c(s))+\delta g^{\prime}(s)[p-c(g(s))]\right)<0$ for all $s$.

Under Assumption 1 there is a unique optimal escapement. The optimal harvest policy, $h^{*}$, is

$$
h^{*}(s)=\left\{\begin{array}{cc}
s-z^{*} & \text { if } \quad s-z^{*} \geq 0 \\
0 & \text { otherwise. }
\end{array}\right.
$$

For cases where $s_{0} \geq z^{*}$, the value function is

$$
\begin{aligned}
\bar{V}\left(s_{0}\right) & =\int_{z^{*}}^{s_{0}}[p-c(\omega)] d \omega+\delta \bar{V}\left(g\left(z^{*}\right)\right) \\
& =\int_{z^{*}}^{s_{0}}[p-c(\omega)] d \omega+\frac{\delta}{1-\delta} \int_{z^{*}}^{g\left(z^{*}\right)}[p-c(\omega)] d \omega=w\left(s_{0}\right)+\frac{\delta}{1-\delta} w\left(g\left(z^{*}\right)\right),
\end{aligned}
$$

where $w(s) \equiv \int_{z^{*}}^{s}[p-c(\omega)] d \omega$ for $s \geq z^{*} . \quad$ When $s_{0}<z^{*}$, the optimal solution requires no harvest until stock has increased above $\mathrm{z}^{*}$. Define $T\left(s_{0}\right)$ by

$$
T\left(s_{0}\right) \equiv \min \left\{t \mid s_{t} \geq z^{*}, h_{l}=0 \text { for all } l \geq 0, s_{l}=g\left(s_{l-1}\right) \text { for all } l \geq 1\right\},
$$


i.e. the minimum number of iterations of the growth function $g($.$) with s_{t}=g\left(g\left(\ldots g\left(s_{0}\right) \ldots\right)\right) \geq z^{*}$.

Then the value function is given by

$$
V\left(s_{0}\right)=\left\{\begin{array}{cl}
\bar{V}\left(s_{0}\right) & \text { if } s_{0} \geq z^{*}, \\
\delta^{T\left(s_{0}\right)} \bar{V}\left(s_{T\left(s_{0}\right)}\right) & \text { if } s_{0}<z^{*}
\end{array}\right.
$$

where $s_{T\left(s_{0}\right)}$ is the stock size after $T\left(s_{0}\right)$ periods of growth starting from stock size $s_{0}$.

We assume that the cooperative solution among $N$ players involves an equal division of harvest among the players: $h_{i}^{*}\left(s_{0}\right) \equiv h^{*}\left(s_{0}\right) / N$, for all $i$. Let $v=V / N$ be the individual payoff under cooperation.

\section{Characterizing a subgame perfect strategy profile}

\subsection{A two-part punishment scheme to support cooperation}

Here we propose a history-dependent strategy profile $\sigma^{*}$ that supports a cooperative outcome and uses a two-part punishment scheme that generates a worst perfect equilibrium once a player has defected from the cooperative outcome. Reversion to a worst perfect equilibrium when a player cheats yields the greatest possible punishment, which in turn gives the greatest incentive not to cheat. Note that a player can always set $h_{i t}=0$ for all $t$ yielding a payoff of 0 . An equilibrium punishment strategy cannot force a player to earn present value negative payoffs, though losses in some periods are possible if offset by positive payoffs in other periods.

Strategy profile $\sigma^{*}$ :

Phase I In period 0, each player $i$ plays $h_{i}^{*}\left(s_{0}\right) \equiv h^{*}\left(s_{0}\right) / N$. If $z_{0}<s_{0}-h^{*}\left(s_{0}\right)$, with resulting stock $s_{1}=g\left(z_{0}\right)$, then move to phase $\operatorname{II}\left(s_{t}\right)$ in period 1 . In period 
$\mathrm{t}=1,2, \ldots$, each player $i$ plays $h_{i}^{*}\left(s_{t}\right) \equiv h^{*}\left(s_{t}\right) / N$ as long as $z_{t-1} \geq s_{t-1}-h^{*}\left(s_{t-1}\right) \quad$ (that is, as long as the stock is equal to or above the level induced by $\left.h^{*}\right)$. If $z_{t-1}<s_{t-1}-h^{*}\left(s_{t-1}\right)$, with resulting stock $s_{t}=g\left(z_{t-1}\right)$, then move to phase $\mathrm{II}\left(s_{t}\right)$ in period $t$.

Phase II $\left(s_{t}\right) \quad$ Each player $i$ plays action $h_{i}^{P}\left(s_{t}\right)$ defined below, resulting in escapement of $z_{t}^{P}\left(s_{t}\right)$. If escapement in period $t$ equals $z_{t}^{P}\left(s_{t}\right)$, then move back to Phase I. If escapement in period $t$ does not equal $z_{t}^{P}\left(s_{t}\right)$, with resulting stock $s_{t+1}$, then begin Phase $\mathrm{II}\left(s_{t+1}\right)$ in the following period $t+1$.

Once a player over-harvests the resource, all players conduct phase II actions where everyone over-extracts the resource to a point where all players incur one-period negative profits. Each player has an incentive to absorb losses in this period because then the resource stock is allowed to recover, players resume cooperation, and each player earns positive returns. Not carrying out the punishment means that phase II will start again in the following period. These two parts of punishment (severe punishment, recovery) can be combined so that each player earns present value returns of zero (the worst perfect equilibrium payoff) evaluated from the beginning of the punishment phase.

We define the punishment strategy starting in period $\tau$ to be consistent with the worst perfect equilibrium starting from the punishment phase. The lowest present value payoff (minmax value) for this game is 0 . Define $h_{i}^{P}\left(s_{\tau}\right) \equiv h^{P}\left(s_{\tau}\right) / N$ such that a player will earn zero present value profit by playing this action in period $\tau$ followed by playing $h_{i}^{*}(s)$ thereafter, 
assuming that the other players play according to the equilibrium strategy:

$$
\frac{1}{N} \int_{s_{\tau}-h^{P}\left(s_{\tau}\right)}^{s_{\tau}}[p-c(\omega)] d \omega+\delta v\left(g\left(z_{\tau}^{P}\left(s_{\tau}\right)\right)\right)=0
$$

where $z_{\tau}^{P}\left(s_{\tau}\right)=s_{\tau}-h^{P}\left(s_{\tau}\right)$, and $v\left(g\left(z_{\tau}^{P}\left(s_{\tau}\right)\right)\right)$ is a player's present value of profit from the cooperative solution starting with $g\left(z_{\tau}^{P}\left(s_{\tau}\right)\right)$ amount of stock. Under the assumptions on the cost function, $c$, there will exist a phase II harvest $h^{P}\left(s_{\tau}\right)$ that satisfies (1) for any stock level $s_{\tau}$. The first term in equation (1) is equal to

$$
\frac{1}{N} \underset{s_{\tau}-[(N-1) / N] h^{P}\left(s_{\tau}\right)}{\int_{\tau}^{s_{\tau}}[p-c(\omega)] d \omega+\frac{1}{N}} \int_{s_{\tau}-h^{P}\left(s_{\tau}\right)}^{\int_{\tau}-[(N-1) / N] h^{P}\left(s_{\tau}\right)}[p-c(\omega)] d \omega .
$$

This expression must be negative for equation (1) to be satisfied. We will assume that the first term is nonpositive.

Assumption 2. $P\left(s_{\tau}\right) \equiv \int_{s_{\tau}-[(N-1) / N] h^{P}\left(s_{\tau}\right)}^{s_{\tau}}[p-c(\omega)] d \omega \leq 0$ for all $s_{\tau}>\underline{s}$.

When Assumption 2 holds, a player cannot deviate from the punishment strategy and earn positive profits during the harsh punishment phase. We discuss when this condition is likely to hold in section 4.3.

We find conditions under which no player is better off by any one-shot deviation from Phases I and II at any stock level. Because each player's period-wise return is bounded from above and the discount rate is positive, the principle of optimality for discounted dynamic programming applies. Therefore, in order to prove that $\sigma^{*}$ is subgame perfect, it is sufficient to show that any one-shot deviation cannot be payoff-improving for any player (Fudenberg and Tirole 1991). Because this is a dynamic game, we need to verify that no player has an incentive 
to deviate from the prescribed strategy in any phase and under any possible stock level. ${ }^{3}$

Given stock $s$, each player who follows cooperation in Phase I earns $v(s)$. If player $i$ deviates from $h_{i}^{*}(s)$, then the maximum the player can obtain is (given zero continuation payoff upon detection of cheating)

$$
\pi(s) \equiv \max _{h_{i} \geq 0} r_{i}\left(h_{i}, h_{-i}^{*}(s), s\right)=\max _{h_{i} \geq 0} \frac{h_{i}}{\sum_{j \neq i} h_{j}^{*}(s)+h_{i}} \int_{s-\sum_{j \neq i} h_{j}^{*}(s)-h_{i}}^{s}[p-c(\omega)] d \omega,
$$

where $h_{-i}^{*}(s)=\left(h_{1}^{*}(s), \ldots, h_{i-1}^{*}(s), h_{i+1}^{*}(s), \ldots, h_{N}^{*}(s)\right)$.

We now show that $\sigma^{*}$ is a subgame perfect equilibrium under certain conditions.

Under Assumptions 1 and 2, the following condition is sufficient to show that $\sigma^{*}$ is subgame perfect.

Assumption 3. $v(K) \geq \pi(K)$, where $K$ is the carrying capacity of the renewable resource.

Assumption 3 states that the present value payoffs from following cooperative strategy must be greater than or equal to the maximum one period deviation payoffs when stock at the beginning of the period equals the carrying capacity, $K$. In what follows we show that no player can gain by deviating in phase I given any current stock level, when Assumption 3 holds. Then we show that no player can gain by deviating in phase II given any stock level.

\subsection{Sustaining cooperation in phase I}

We first show that no player can gain by deviating in phase I at all initial stock levels, $s \in(0, K]$.

Beginning with stock $s$, each player earns $v(s)$ by playing $\sigma^{*}$. If player $i$ deviates in phase I,

\footnotetext{
${ }^{3}$ See Dutta (1995a, b) and Polasky et al. (2006) for a similar analysis in a dynamic game context.
} 
then the player earns at most $\pi(s)$ in the present period. Starting in phase II, player i earns present value payoffs of 0 . Hence, player $i$ has no incentive to deviate in phase I if the following condition holds:

$$
v(s) \geq \pi(s) \text { for all } s .
$$

The following three lemmas will show that $v(K) \geq \pi(K)$ is sufficient for the above inequality to hold.

Let $h^{D}(s)$ be the harvest that maximizes the payoff upon deviation when stock size is $s$ where the other players choose $h_{-i}^{*}(s)$. Lemma 1 characterizes $h^{D}(s)$.

Lemma 1. The optimal deviation $h^{D}(s)$ satisfies

$$
h^{D}(s)\left\{\begin{array}{cc}
=0 & \text { if } s \in(0, \underline{s}], \\
=s-\sum_{j \neq i} h_{j}^{*}(s)-\underline{s} & \text { if } s \in\left(\underline{s}, z^{*}\right], \\
>s-\sum_{j \neq i} h_{j}^{*}(s)-\underline{s} & \text { if } s \in\left(z^{*}, K\right] .
\end{array}\right.
$$

See the Appendix for the proof. Lemma 1 states that the optimal deviation in period $t$ given $s>\underline{s}$ is to extract the resource to a point at or below $\underline{s}$. The intuition for the result is that at $\underline{s}$ the unit rent is zero. With further extraction, the total return decreases but the player's share of total returns increases. If other players are harvesting, it is therefore optimal for a deviator to harvest so that the resulting resource stock is less than $\underline{s}$. Lemma 1 implies the following about the slopes of $\pi$ and $v$.

Lemma 2. $\pi^{\prime}(s)>v^{\prime}(s)$ for all $s \in\left[z^{*}, K\right)$.

See the Appendix for the proof. Lemma 2 implies that $\pi$ decreases faster than $v$ as stock decreases from $K$ to some level $s \geq z^{*}$. This implies that $v(s) \geq \pi(s)$ for all $s \in\left[z^{*}, K\right)$ if 
$v(K) \geq \pi(K)$

For $v(K) \geq \pi(K)$ to imply inequality (2), it remains to show that $v(s) \geq \pi(s)$ for all $s \in\left(\underline{s}, z^{*}\right) \quad$ whenever $v\left(z^{*}\right) \geq \pi\left(z^{*}\right)$. Lemma 3 addresses this last point.

Lemma 3. $v^{\prime \prime}(s)-\pi^{\prime \prime}(s)<0$ holds for any $s \in\left(\underline{s}, z^{*}\right)$.

See the Appendix for the proof.

Lemma 4. If $v(K) \geq \pi(K)$, then $v(s) \geq \pi(s)$ for all $s \in[s, K)$.

Proof. It follows from Lemma 3, $v^{\prime}(\underline{s})>\pi^{\prime}(\underline{s})$ and $v^{\prime}\left(z^{*}\right)<\pi^{\prime}\left(z^{*}\right)$ that there exists unique $\hat{s} \in\left(\underline{s}, z^{*}\right)$ such that

(i) $\pi^{\prime}(\hat{s})=v^{\prime}(\hat{s})$,

(ii) $\pi^{\prime}(s)>v^{\prime}(s)$ if $\hat{s}<s \leq z^{*}$ and

(iii) $\pi^{\prime}(s)<v^{\prime}(s)$ if $\underline{s} \leq s<\hat{s}$.

Together with the fact that $v(\underline{s})>\pi(\underline{s})$, the assumption that $v(K) \geq \pi(K)$, and Lemma 2 , we conclude that $v(s) \geq \pi(s)$ for all $s \leq K$.

Since, $v(s) \geq \pi(s)$ for all $s$, no player would find it profitable to deviate from Phase I.

Figure 1 describes an example where $v(K) \geq \pi(K)$ holds. The figure is based on a unit cost function $c(s)=1 / s$ and a logistic growth function $g(s)=s+r s(1-s / K)$ where $r<1$ is the intrinsic growth rate of the resource. As in Lemma 4, $v(K) \geq \pi(K)$ implies $v(s) \geq \pi(s)$ for all $0<s \leq K$.

[Figure 1] 


\subsection{Supporting cooperation in phase II}

Now we show that players cannot gain by deviating in phase $\mathrm{II}(s)$ for any post-deviation stock level $s$.

Lemma 5. Under Assumption 2, no player has an incentive to deviate in phase II(s) for all s.

Proof. By following the equilibrium strategy in Phase $\mathrm{II}(s)$, a player earns 0 present value returns. In the first period of phase $\operatorname{II}(s)$, the maximum payoff player $i$ can earn upon deviation is given by

$$
\max _{h_{i} \geq 0} \frac{h_{i}}{h_{i}+\sum_{j \neq i} h_{j}^{P}(s)} \int_{s-h_{i}-\sum_{j \neq i}^{s} h_{j}^{P}(s)}^{s}[p-c(\omega)] d \omega .
$$

Under Assumption 2, $\quad \int_{s-\sum_{j \neq i} h_{j}^{p}(s)}^{s}[p-c(\omega)] d \omega \leq 0$ for all $s>\underline{s}$ (and is certainly negative for $s<\underline{s}$ ). Therefore, there is no positive $h_{i}$ that can generate a positive return when other players play according to the punishment strategy: $h_{j}=h_{j}^{p}$ for $j \neq i$. A deviation against the punishment triggers the start of a new punishment phase in which all players earn present value profits of zero. Therefore, there is no profitable deviation in the severe punishment phase (the period following a defection from the cooperative strategy). If there is no deviation in the first period of phase II $(s)$, players then revert back to phase I, for which we have already demonstrated that no profitable deviations exist. Hence, under Assumption $2(P(s) \leq 0$ for all $s>\underline{s})$, there are no profitable unilateral deviations for any level of stock.

Assumption 2 is likely to hold when $N$ is not too small or growth of the stock is not too large. Because the optimal deviation drives escapement below $\underline{s}$, as long as stock growth is not too fast, stock may be either lower than $\underline{s}$ or else not far above. With large $N$, any one player's share of 
harvest is small making it unlikely that even if this player harvests nothing that escapement will be above $\underline{s}$.

\subsection{Summary}

Lemmas 1-5 imply that there is no profitable deviation against strategy $\mathrm{s}^{*}$ in either phase I or phase II. We summarize the previous analysis in the following proposition.

Proposition 1. Under Assumptions 1, 2, and 3, $\sigma^{*}$ is a subgame perfect equilibrium and supports a cooperative (first best) outcome.

\section{Numerical Illustration}

We use a numerical example to illustrate the results of the previous section. We assume a unit cost function $c(s)=1 / s$ and a logistic growth function $g(s)=s+r s(1-s / K)$ where $r<1$. We describe when the sufficient conditions for Proposition 1 hold.

Figure 2 shows the combinations for the number of players, $N$, and the discount factor, $\delta$, for which Assumption 3 and Assumption 2 hold. Assumption 3 states that the present value of the cooperative strategy exceeds the value of deviation for a player when the stock level begins at the unexploited carrying capacity level of stock, $K$. In the figure, any combination of $N$ and $\delta$ above (below) the line with square markers satisfies (does not satisfy) Assumption 3. Assumption 2 states that a player cannot earn positive profits during the punishment phase $(P(s) \leq 0)$. Any combination of $N$ and $\delta$ above (below) the line with triangle markers satisfies (does not satisfy) Assumption 2. For $N \geq 4$, Assumption 3 is the more binding constraint (i.e., holds for a smaller range of discount factors). As shown in the figure, Assumption 3 holds for a 
smaller range of discount factors as $N$ grows. In other words, it becomes more difficult to support cooperation as $N$ grows. This is the standard finding in the literature. On the other hand, for small $N,(N=3$ in the case shown in Figure 2), Assumption 2 is the more binding constraint. Because Assumption 2 holds for a wider range of values of discount factors as $N$ gets larger, it may be possible to support cooperation as a subgame perfect equilibrium outcome for a larger $N$ even though it is not possible to do so for smaller $N$. This counter-intuitive result may occur because having more players makes it easier to ensure that players cannot deviate against the punishment strategy, which is necessary to ensure that the strategies constitute a subgame perfect equilibrium.

Figure 3 draws the value of function $P(s)$ defined in Assumption 2 given optimal phase-I deviations. This value must be nonpositive in order for the phase-II strategy to be a best response for each player. With $\delta=0.9$, Assumption 2 holds with $N=4$ but not with $N=3$.

[Figure 2, Figure 3]

The implication is that the range of potential punishments increases when group size increases from 3 to 4 . This increase in $N$ reduces the degree to which any one individual must increase harvest to inflict the punishment, which tends to lower the cost associated with following through with the punishment. As such, larger punishments become credible, which tends to make cooperation easier to support. But increases in group size raise the potential gains from defection: as $N$ grows, each player is accorded a smaller share of the cooperative pie, which increases the potential one-time gain from defection. Our simulation results indicate that, for the particular example we analyze, this effect becomes more important than the enhanced 
credibility of punishments for values of $N$ at or above 4 . On balance, we see a non-monotonic effect of group size upon cooperative tendencies. It is interesting to compare this result to the findings in Mason and Phillips (1997). In experiments based on a common-property resource with groups of 2, 3, 4 and 5 players, they found the most cooperative behavior occurred with an intermediate group size of 3, rather than at the lowest group size (2) or highest group size (5).

For a smaller intrinsic resource growth rate $r$, Assumption 2 holds with lower discount factors but Assumption 3 holds with only higher discount factors. In Polasky et al. (2006) with observable actions, an increase in resource growth rate was favorable to supporting a cooperative outcome. With unobservable actions, players have a stronger incentive to deviate from phase II when the resource grows faster.

If Assumption 2 does not hold and $P(s)>0$ for some stock levels, then each player may be better off by deviating in phase II of $\sigma^{*}$. In this case, the worst perfect equilibrium payoff will be positive. Hence, by deviating in phase I a player can receive a positive one-shot return as well as positive present-value returns starting from the next period. Phase II must be redefined so that the payoffs upon deviation in phase II is lower than the positive worst perfect equilibrium payoff $w>0$. The condition for phase I then becomes $v(s) \geq \pi(s)+\delta w$ for all $s$.

\section{Discussion}

We analyzed cooperation in common-property resource use in a discrete-time framework with non-linear stock dependent costs and imperfect monitoring of resource users' harvests. Given unobservable actions, punishment to deter over-harvesting involves symmetric punishment of all resource users. This paper proposed a strategy profile with symmetric two-part punishment where, upon someone's deviation from cooperation, every resource user over-harvests the 
resource for one period, followed by reestablishment of cooperative harvesting. This two-part punishment scheme can be designed so that players receive the worst perfect equilibrium payoffs following a defection. We found conditions under which such a strategy is a subgame perfect equilibrium supporting cooperation. Unlike the case of observable actions (Polasky et al. 2006), cooperation is not necessarily supportable when the number of resource users is small, when the resource grows faster, or when the players have a larger discount factor.

When each player's action is observable to others, then the players can apply a punishment specific to the player who deviated from cooperation. In this case, the players who did not cheat can receive positive payoffs during the punishment phase, with only the player who did cheat subject to the worst perfect equilibrium payoffs. Polasky et al. (2006) analyzes a continuous-time version of a dynamic game of common property resources with observable actions.

The simple punishment strategy proposed in this paper, severe group punishment following a defection followed by forgiveness and a return to cooperation, has several attractive features. First, as we have emphasized through the paper, such a strategy can be designed to generate the strongest possible punishment (worst perfect equilibrium), which gives the greatest possible deterrent to defection.

Second, such a strategy is consistent with empirical evidence on how people behave in common property and public goods games. In such situations, people appear to behave in a reciprocal manner, meaning that they are nice to those who cooperate but quite willing to inflict punishment on those who fail to be cooperative, even when such punishment is costly to themselves (Fehr and Gachter, 2000a, 2000b, 2002). Ostrom (2000) summarizes typical behavior observed in common property and public goods games as players being "conditional cooperators" 
and "willing punishers." It seems likely that people who are supposed to cooperate in harvesting a common property resource would be willing to exact some revenge immediately following learning that someone in the community has cheated. Whether they would go as far as we have posited in specifying the worst-perfect equilibrium strategy is questionable. However, even lesser punishments, willingly inflicted immediately following cheating, may be sufficient in some circumstances to deter defection from the cooperative strategy.

Finally, the two-part punishment strategy, unlike a simple single-part punishment strategy such as a simple trigger strategy like that used in Hannesson (1997), is relatively immune to renegotiation. The only period in which payoffs are not on the Pareto frontier is the period immediately following a defection, when players probably are willing to inflict costly punishment. As long as the group "takes its medicine" by going through with the punishment, cooperation is reestablished and payoffs are once again on the Pareto frontier, and hence, not subject to renegotiation pressure. In contrast, a simple single-part punishment strategy involves dooming players to suboptimal payoffs forever following a defection. This fact raises questions about why players would not at some future time find in their best interests to forgive and forget, i.e., renegotiate to get back to the Pareto frontier. But, of course, if they do so, this will call into question the deterrence value of the single-part punishment, which in turn may cause cooperation to fail.

In this paper, we assumed deterministic resource transitions and perfect monitoring of stock. In fisheries, and other renewable resources, unexpected fluctuations in stock due to random environmental events are common as is imprecise measurement of stock levels (Roughgarden and Smith 1996; Sethi et al. 2005). One can extend previous studies on repeated oligopoly game with random demand (Abreu et al. 1986; Green and Porter 1984) to the common 
property resource situation with stochastic growth or imprecise measurement. While analyzing an N-player model with uncertain stock would present formidable technical challenges, it is clearly an interesting direction for future work.

\section{Appendix}

\section{Uniqueness of the optimal harvesting rule}

The first best outcome is given by a solution to the following problem.

$$
\begin{aligned}
& \max _{\substack{\left\{h_{t}, x=0 \\
x_{t}\right.}} \sum_{t=0}^{\infty} \delta^{t} \int_{s_{t}-h_{t}}^{s_{t}}[p-c(\omega)] d \omega \\
& \text { s.t. } \quad s_{t+1}=g\left(s_{t}-h_{t}\right) \quad t=0,1, \ldots, h_{t} \geq 0 \quad t=0,1, \ldots \text { given } s_{0} \in(0, K] .
\end{aligned}
$$

Letting $z_{t}=s_{t}-h_{t}$, the first order condition is

$$
\begin{gathered}
-\left[p-c\left(z_{t}\right)\right]+\delta g^{\prime}\left(z_{t}\right)\left[P-c\left(g\left(z_{t}\right)\right)\right]=0 \text { if } z_{t} \leq s_{t}, \\
\text { and } z_{t}=s_{t} \text { if } z^{*}>s_{t} \text { for all } t .
\end{gathered}
$$

That is, the optimal recruitment given the beginning-of-period-stock $s_{t}$ is independent of $s_{t}$. The existence of a solution $z^{*}$ follows from

$$
\begin{gathered}
-[p-c(\underline{s})]+\delta g^{\prime}(\underline{s})[p-c(g(\underline{s}))]>0 \quad\left(g^{\prime}>0, g(s)>s \text { and } c^{\prime}<0\right), \\
-[p-c(K)]+\delta g^{\prime}(K)[p-c(g(K))]<0 \quad\left(g^{\prime}(K)<1, g(K)=K \text { and } \delta<1\right)
\end{gathered}
$$

and the continuity of $c$ and $g$. A sufficient condition for the uniqueness of the solution $\mathrm{z}^{*}$ is that the second-order derivative is negative:

$$
\text { (*) } c^{\prime}(z)+\delta g^{\prime \prime}(z)[p-c(g(z))]-\delta c^{\prime}(g(z))\left[g^{\prime}(z)\right]^{2}<0 \quad \text { for all } z \in(\underline{s}, K) .
$$

The first two terms are negative and the third term is positive, so the sign is indeterminate. This condition holds, for example, if $c(s)=1 / s$ and $g(s)=s^{\alpha}$ with $0<\alpha<1$. In this case, the first-order condition is $-\left[p-1 / z_{t}\right]+\delta \alpha z_{t}{ }^{\alpha-1}\left[p-1 /\left(z_{t}^{\alpha}\right)\right]=-p+(1-\delta \alpha) z_{t}{ }^{-1}+p \delta \alpha z_{t}{ }^{\alpha-1}=0 \quad$ and the second-order derivative is $-(1-\delta \alpha) z_{t}^{-2}+p \delta \alpha(\alpha-1) z_{t}{ }^{\alpha-2}<0$ for all $z_{t}$. Condition (*) also holds if $c(s)=1 / s$ and $g(s)=s+r s(1-s / K)$ with $r \in(0,1)$ and $K>0$. In this case, the first order condition is $-p+\frac{1-\delta}{z_{t}}+\delta p\left(1+r-\frac{2 r z_{t}}{K}\right)+\frac{\delta r}{K(1+r)-r z_{t}}=0$ and the second order 
condition is

$$
-\frac{1-\delta}{z_{t}^{2}}-\frac{\delta r\left[2 p K\left(1+r-\frac{r z_{t}}{K}\right)^{2}-r\right]}{\left[K(1+r)-r z_{t}\right]^{2}}<0
$$

which always holds because $p>c(K)=1 / K$ (and hence the expression inside the square bracket is positive).

\section{Proof of Lemma 1.}

The first order condition for $h^{D}(s)$ is

$$
\frac{\partial}{\partial h_{i}} r_{i}\left(h_{i}, h_{-i}^{*}(s), s\right)=\frac{\sum_{j \neq i} h_{j}^{*}(s)}{\left(h_{i}+\sum_{j \neq i} h_{j}^{*}(s)\right)^{2}} \int_{s-\sum_{j \neq i} h_{j}^{*}(s)-h_{i}}^{s}[p-c(\omega)] d \omega+\frac{h_{i}}{\left(h_{i}+\sum_{j \neq i} h_{j}^{*}(s)\right)}\left[p-c\left(s-\sum_{j \neq i} h_{j}^{*}(s)-h_{i}\right)\right] \leq 0 .
$$

Clearly the optimal $h^{D}(s)$ is zero when the stock level is below $\underline{s}$. When $s \in\left(\underline{s}, z^{*}\right]$, we have $h_{j}^{*}(s)=0$ for all $j \neq i$ and hence the above derivative is equal to zero when $s-\sum_{j \neq i} h_{j}^{*}(s)-h_{i}=s-h_{i}=\underline{s}$. If $s \in\left(z^{*}, K\right]$, then $h_{j}^{*}(s)>0$ for all $j$ and hence the above derivative is positive for all $h_{i}$ such that $s-\sum_{j \neq i} h_{j}^{*}(s)-h_{i} \geq \underline{s}$. This means that the optimal unilateral deviation for player $i$ is to harvest so that the stock level is lower than $\underline{S}$.

\section{Proof of Lemma 2.}

For $s \in\left[z^{*}, K\right)$, we have $v^{\prime}(s)=\frac{1}{N}[p-c(s)]$ and

$$
\begin{aligned}
\pi^{\prime}(s) & =\frac{h^{D^{\prime}}(s)\left[H_{-i}^{*}(s)+h^{D}(s)\right]-h^{D}(s)\left[H_{-i}^{*}(s)+h^{D^{\prime}}(s)\right]}{\left[H_{-i}^{*}(s)+h^{D}(s)\right]^{2}} \int_{s-H_{-i}^{*}(s)-h^{D}(s)}^{s}[p-c(\omega)] d \omega \\
+ & \frac{h^{D}(s)}{H_{-i}^{*}(s)+h^{D}(s)}\left[p-c(s)-\left[\left(p-c\left(s-H_{-i}^{*}(s)-h^{D}(s)\right)\right)\left(1-H_{-i}^{*}(s)-h^{D \prime}(s)\right)\right]\right] \\
= & \frac{N-1}{N} \frac{h^{D}(s)}{\left[H_{-i}^{*}(s)+h^{D}(s)\right]^{2}} \int_{s-H_{-i}^{*}(s)-h^{D}(s)}^{s}[p-c(\omega)] d \omega \\
& +\frac{h^{D}(s)}{H_{-i}^{*}(s)+h^{D}(s)}\left[p-c(s)-\left[\left(p-c\left(s-H_{-i}^{*}(s)-h^{D}(s)\right)\right) \frac{1}{N}\right]\right],
\end{aligned}
$$


where $H_{-i}^{*}(s) \equiv \sum_{j \neq i} h_{j}^{*}(s)$ and $h^{D}(s)$ maximizes the payoff upon deviation when stock size is $s$. The second equality follows from the envelope theorem and $H_{-i}^{*}{ }^{\prime}(s)=\frac{N-1}{N}$ for all $s \in\left(z^{*}, K\right)$. In the last expression, the first term is positive because $\int_{s-H_{-i}^{*}(s)-h^{D}(s)}^{s}[p-c(\omega)] d \omega>0$. In the second term, we have $p-c\left(s-H_{-i}^{*}(s)-h^{D}(s)\right)<0$ and $\frac{h^{D}(s)}{H_{-i}^{*}(s)+h^{D}(s)}>\frac{1}{N}$ by Lemma 1. Hence, $\pi^{\prime}(s)$ is greater than $\frac{1}{N}[p-c(s)]$. These facts imply $\pi^{\prime}(s)>v^{\prime}(s)$ for $s \in\left[z^{*}, K\right)$.

\section{Proof of Lemma 3.}

For all $s \in\left(\underline{s}, z^{*}\right)$ we have $\pi(s)=\int_{\underline{s}}^{s}[p-c(w)] d w$. The functional form of $v$ on $\left(\underline{s}, z^{*}\right)$ depends on the value $T(\underline{s})$, that is, the number of periods the players should choose zero harvest on the efficient path. We will show that $v^{\prime}(s)-\pi^{\prime}(s)$ is strictly decreasing for an arbitrary value of $T(\underline{s})$. First, consider stock level $s<z^{*}$ which is sufficiently close to $z^{*}$ such that $g(s)>z^{*}$. We have $T(s)=1$ and hence $v(s)=\frac{\delta}{N} \int_{z^{*}}^{g(s)}[p-c(w)] d w+\delta^{2} v\left(g\left(z^{*}\right)\right)$. Then

$$
\begin{aligned}
& v^{\prime}(s)-\pi^{\prime}(s)=\quad-(p-c(s))+\frac{\delta}{N}[p-c(g(s))] g^{\prime}(s) \\
& =-\frac{1}{N}(p-c(s))+\frac{\delta}{N}[p-c(g(s))] g^{\prime}(s)-\frac{N-1}{N}(p-c(s)) \\
& =\quad \frac{1}{N} f(s)-\frac{N-1}{N}(p-c(s)),
\end{aligned}
$$

where $f(s) \equiv-(p-c(s))+\delta g^{\prime}(s)[p-c(g(s))]$. Because $f^{\prime}<0$ by Assumption 1 and $c^{\prime}<0$, we have

$$
v^{\prime \prime}(s)-\pi^{\prime \prime}(s)=\frac{1}{N} f^{\prime}(s)+\frac{N-1}{N} c^{\prime}(s)<0 .
$$

Secondly, suppose $T(\underline{s})>1$. Then, for stock size $s$ such that $g(s)<z^{*}<g(g(s)$ ) (i.e. $T(s)=2$ ), efficiency requires players to choose zero harvest for two periods. Hence, 


$$
v^{\prime}(s)=\frac{\delta^{2}}{N}[p-c(g(g(s)))] g^{\prime}(g(s)) g^{\prime}(s)
$$

and we have

$$
\begin{aligned}
v^{\prime}(s)-\pi^{\prime}(s) & = & -(p-c(s))+\frac{\delta}{N}[p-c(g(s))] g^{\prime}(s)-\frac{\delta}{N}[p-c(g(s))] g^{\prime}(s)+\frac{\delta^{2}}{N}[p-c(g(g(s)))] g^{\prime}(g(s)) g^{\prime}(s) \\
& = & \frac{1}{N} f(s)-\frac{N-1}{N}(p-c(s))+\frac{\delta}{N} g^{\prime}(s)\left[-[p-c(g(s))]+[p-c(g(g(s)))] g^{\prime}(g(s))\right] \\
& = & \frac{1}{N} f(s)-\frac{N-1}{N}(p-c(s))+\frac{\delta}{N} g^{\prime}(s) f(g(s)) .
\end{aligned}
$$

Hence,

$$
v^{\prime \prime}(s)-\pi^{\prime \prime}(s)=\frac{1}{N} f^{\prime}(s)+\frac{N-1}{N} c^{\prime}(s)+\frac{\delta}{N}\left[g^{\prime \prime}(s) f(g(s))+g^{\prime}(s) f^{\prime}(g(s)) g^{\prime}(s)\right]<0
$$

because $g^{\prime}>0, g^{\prime \prime}<0, f^{\prime}<0$ and $f(s)>0$ for all $s<z^{*}$.

Similarly, suppose $T(\underline{s})>2$ and consider stock $s>\underline{s}$ such that $g(g(s))<z^{*}<g(g(g(s)))$.

We have

$$
\begin{aligned}
v^{\prime}(s)-\pi^{\prime}(s)= & -(p-c(s))+\frac{\delta}{N}[p-c(g(s))] g^{\prime}(s)-\frac{\delta}{N}[p-c(g(s))] g^{\prime}(s)+\frac{\delta^{2}}{N}[p-c(g(g(s)))] g^{\prime}(g(s)) g^{\prime}(s) \\
& -\frac{\delta^{2}}{N}[p-c(g(g(s)))] g^{\prime}(g(s)) g^{\prime}(s)+\frac{\delta^{3}}{N}[p-c(g(g(g(s))))] g^{\prime}(g(g(s))) g^{\prime}(g(s)) g^{\prime}(s) \\
= & \frac{1}{N} f(s)-\frac{N-1}{N}(p-c(s))+\frac{\delta}{N} g^{\prime}(s) f(g(s))+\frac{\delta^{2}}{N} g^{\prime}(s) g^{\prime}(g(s)) f(g(g(s)) .
\end{aligned}
$$

It then follows that

$$
v^{\prime \prime}(s)-\pi^{\prime \prime}(s)=\frac{1}{N} f^{\prime}+\frac{N-1}{N} c^{\prime}+\frac{\delta}{N}\left(g^{\prime \prime} f+g^{\prime} f^{\prime} g^{\prime}\right)+\frac{\delta^{2}}{N}\left[\left(g^{\prime \prime} g^{\prime}+g^{\prime} g^{\prime \prime} g^{\prime}\right) f+g^{\prime} g^{\prime} f^{\prime}\right]<0
$$

(the arguments of functions $f$ and $g$ are omitted). The same argument holds for any $s \in\left(\underline{s}, z^{*}\right)$ given any value of $T(\underline{s})$. Hence, $v^{\prime \prime}(s)-\pi^{\prime \prime}(s)<0$ holds for any $s \in\left(\underline{s}, z^{*}\right)$. 


\section{References}

Abreu, D. (1988) “On the Theory of Infinitely Repeated Games with Discounting” Econometrica 56, 383 - 396.

Abreu, D., Pearce, D., and E. S. Stacchetti (1986) “Optimal Cartel Equilibria with Imperfect Monitoring” Journal of Economic Theory 39, 251 - 269.

Abreu, D., D. Pearce and E. S. Stacchetti (1990) “Toward a Theory of Discounted Repeated Games with Imperfect Monitoring” Econometrica 58, 1041 - 1063.

Benhabib, J. and R. Radner (1992) “The Joint Exploitation of a Productive Asset: A Game-theoretic Approach” Economic Theory 2, 155 - 190.

Brown, G. (1974) “An Optimal Program for Managing Common Property Resources with Congestion Externalities” Journal of Political Economy 82, 163 - 173.

Casari, M. and C. R. Plott (2003) “Decentralized Management of Common Property Resources: Experiments with a Centuries-old Institution” Journal of Economic Behavior \& Organization 51, $217-247$.

Cason, T. N. and F. U. Khan (1999) “A Laboratory Study of Voluntary Public Goods Provision with Imperfect Monitoring And Communication” Journal of Development Economics 58, $533-552$.

Cave, J. (1987) “Long-Term Competition in a Dynamic Game: The Cold Fish War” The RAND Journal of Economics 18, 596 - 610.

Chermak, J. M. and K. Krause (2002): “Individual Response, Information, and Intergenerational Common Pool Problems” Journal of Environmental Economics and Management 43, 47 -70 . 
Clark, C.W. (1973), "Profit Maximization and the Extinction of Animal Species” Journal of Political Economy 81(4): 950-961.

Dutta, P. K. (1995a) “A Folk Theorem for Stochastic Games” Journal of Economic Theory 66, $1-32$.

Dutta, P. K. (1995b) “Collusion, Discounting and Dynamic Games” Journal of Economic Theory 66, $289-306$.

Dutta, P. K. and R. K. Sundaram (1993) “The Tragedy of the Commons?” Economic Theory 3, $413-26$.

Feeny, D., S. Hanna and A. F. McEvoy (1996) "Questioning the Assumptions of the 'Tragedy of the Commons’ Model of Fisheries” Land Economics 72, 187-205.

Fehr, E. and S. Gachter (2000a) “Cooperation and Punishment in Public Goods Experiments” American Economic Review 90, 80 - 994.

Fehr, E. and S. Gachter (2000b) "Fairness and Retaliation: The Economics of Reciprocity" Journal of Economic Perspectives 14(3), 159-81.

Fehr, E. and S. Gachter (2002) “Altruistic Punishment in Humans” Nature 415, 137-40.

Fudenberg, D. and J. Tirole (1991) Game Theory, MIT Press, Cambridge, MA.

Gordon, H. S. (1954) “The Economic Theory of a Common-Property Resource: The Fishery” Journal of Political Economy 62, $124-142$.

Green, E. J. and R. H. Porter (1984) “Noncooperative Collusion under Imperfect Price Information” Econometrica 52, 87 - 100.

Hackett, S., E. Schlager and J. Walker (1994) "The Role of Communication in Resolving Commons Dilemmas: Experimental Evidence with Heterogeneous Appropriators” Journal of Environmental Economics and Management 27, 99 - 126. 
Hannesson, R. (1997) "Fishing as a Supergame” Journal of Environmental Economics and Management 32, $309-322$.

Hardin, G. (1968) “The Tragedy of the Commons” Science 162, 1243-48.

Markets and Rent Dissipation in Regulated Open Access Fisheries

Homans, F.R. and J. E. Wilen (1997) "A Model of Regulated Open Access Resource Use” Journal of Environmental Economics and Management 32, 1-21.

Isaac, R. M. and J. M. Walker (1988) "Communication and Free-Riding: The Voluntary Contribution Mechanism” Economic Inquiry 26, 585 - 605.

Laukkanen, M. (2003) “Cooperative and Non-cooperative Harvesting in a Stochastic Sequential Fishery” Journal of Environmental Economics and Management 45, 454-473.

Ledyard, J.O. (1995) “Public Good: A Survey of Experimental Research” in The Handbook of Experimental Economics, J. H. Kagel and A. E. Roth, Eds. Princeton University Press.

Levhari, D. and L. J. Mirman (1980) "The Great Fish War: An Example Using a Dynamic Cournot-Nash Solution” Bell Journal of Economics 11, 322 - 334.

Mason, C. F. and O. R. Philips (1997) "Mitigating the Tragedy of the Commons through Cooperation: An Experimental Evaluation” Journal of Environmental Economics and Management 34, 148 - 172.

Mason, C. F. and S. Polasky (1997) "The Optimal Number of Firms in the Commons: A Dynamic Approach” Canadian Journal of Economics 30, 1143 - 1160.

Ostrom, E. (1990) Governing the Commons, Cambridge University Press, Cambridge, UK.

Ostrom, E. (2000) “Collective Action and the Evolution of Social Norms” Journal of Economic Perspectives 14(3), 137-58.

Ostrom E, R. Gardner, and J. Walker (1994) Rules, Games, and Common Pool Resources 
University of Michigan Press, Ann Arbor.

Ostrom, E., Walker, J. M. and R. Gardner (1992) "Covenants With and Without A Sword: Self-Governance is Possible” American Political Science Review, 86, 404 - 417.

Polasky, S, N. Tarui, G. M. Ellis and C. F. Mason (2006) "Cooperation in the Commons" Economic Theory, 29, 71 - 88.

Roughgarden, J. and F. Smith (1996) “Why Fisheries Collapse and What To Do About It?” Proceedings of the National Academy of Sciences 93, 5078-5083.

Rustichini, A. (1992) “Second Best Equilibria for Games of Joint Exploitation of a Productive Asset” Economic Theory 2, 191 - 196.

Sadrieh, A. and H. A. A. Verbon (2006) "Inequality, Cooperation, and Growth: An Experimental Study” European Economic Review 50, 1197-1222.

Sethi, G., C. Costello, A. Fisher, M. Hanemann and L. Karp (2005) “Fishery Management under Multiple Uncertainty” Journal of Environmental Economics and Management 50, 300-318. 




Figure 1: Payoffs upon cooperation $(v)$ and deviation $(\pi)$

This figure is based on an example with $r=0.5, K=100, \delta=0.9, p=0.05$, and $N=4$. In this case, we have $\underline{s}=20$ and $z^{*}=51.3$. Function $v$ is the payoff upon cooperation in phase 1 , and function $\pi$ represents the maximum payoff upon deviation from phase $\mathrm{I}$. 


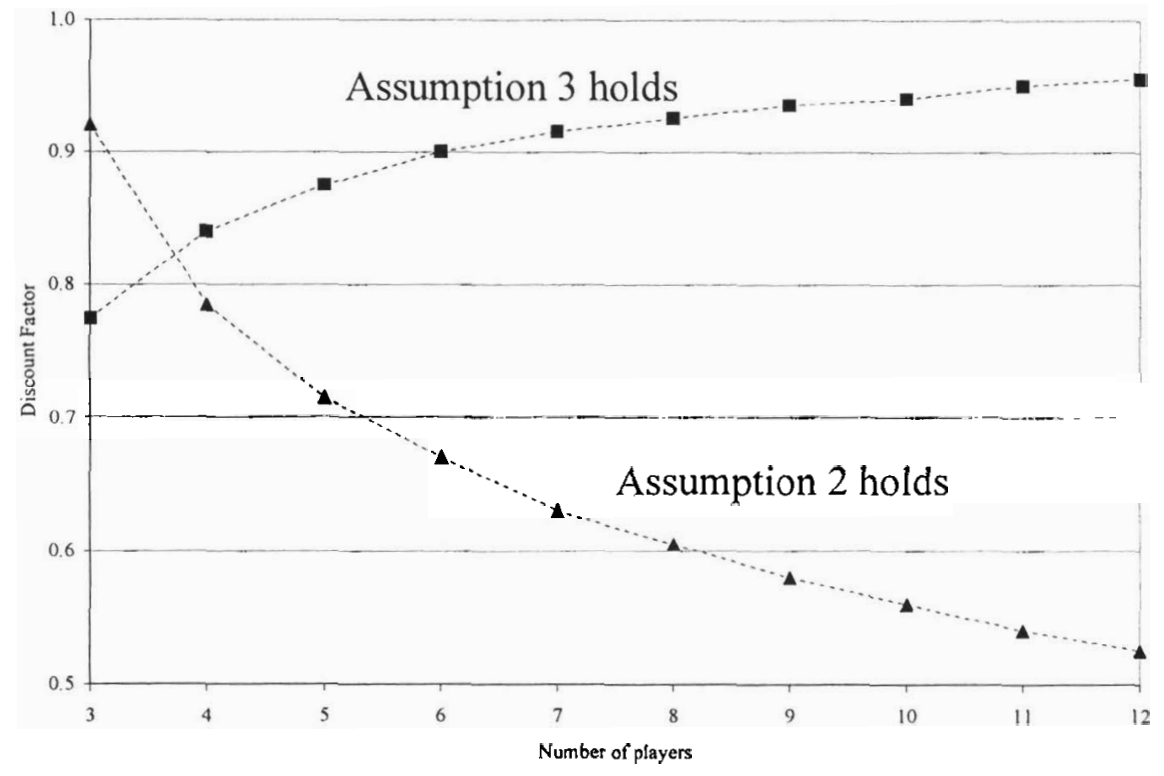

Figure 2: Tradeoff between Assumptions 2 and 3.

This figure is based on an example with $r=0.5, K=100$, and $p=0.05$. Assumption 3 holds in the area above the curve with square markers. Assumption 2 holds in the area above the curve with triangle markers. 


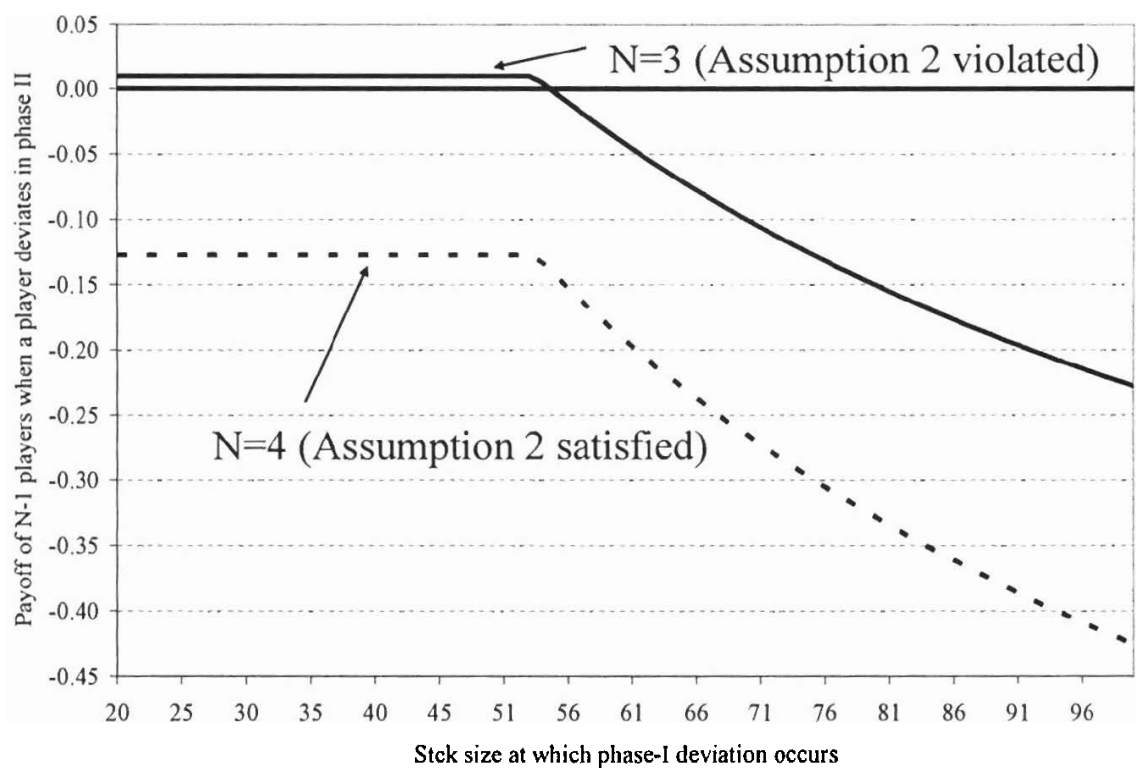

Figure 3: Assumption 2 holds with $N=4$, but not with $N=3$.

This figure is based on an example with $r=0.8, K=100, \delta=0.9$, and $p=0.05$. 